\title{
COVID-19 outpatients and surviving inpatients exhibit comparable blood test results that are distinct from non-surviving inpatients
}

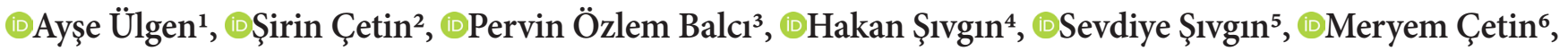 \\ (D)Wentian $\mathrm{Li}^{7}$ \\ ${ }^{1}$ Girne American University, Faculty of Medicine, Department of Biostatistics, Karmi, Girne, Turkish Republic of Northern Cyprus \\ ${ }^{2}$ Gaziosmanpaşa University, Faculty of Medicine, Department of Biostatistics, Tokat, Turkey \\ ${ }^{3}$ Tokat State Hospital, Department of Medical Microbiology, Tokat, Turkey \\ ${ }^{4}$ Tokat State Hospital, Department of Internal Medicine, Tokat, Turkey \\ ${ }^{5}$ Tokat State Hospital, Department of Anesthesiology and Reanimation, Tokat, Turkey \\ ${ }^{6}$ Amasya University, Faculty of Medicine, Department of Medical Microbiology, Amasya, Turkey \\ ${ }^{7}$ The Feinstein Institutes for Medical Research, The Robert S. Boas Center for Genomics and Human Genetics, Northwell Health, Manhasset, NY, USA
}

Cite this article as: Ülgen A, Çetin Ş, Balcı PÖ, et al. COVID-19 outpatients and surviving inpatients exhibit comparable blood test results that are distinct from non-surviving inpatients. J Health Sci Med 2021; 4(3): 306-313.

\begin{abstract}
Aim: The decision of admitting COVID-19 patients as inpatients is mostly determined by chest X-ray based diagnosis of pneumonia severity. However, prognosis of inpatients may diverge into two groups, one group of inpatients did not survive while another group did. Material and Method: More than 100 COVID-19 outpatients are collected from Tokat, Turkey in three categories: outpatients, surviving inpatients, and deceased inpatients. Their blood test profiles are analyzed and compared by dimension reduction techniques and classic statistical tests.

Results: We observe that surviving inpatients share a common blood test profile with the outpatients, whereas non-surviving inpatients are distinctively different. The non-surviving inpatients are on average older. Among patients older than certain age, non-surviving inpatients have higher neutrophil level, lower lymphocyte level (thus higher neutrophil/lymphocyte ratio), lower calcium level, higher C-reactive-protein, sodium, whole blood cell level, andlower hemoglobin level, than the surviving patients (whether these are inpatients or outpatients).

Conclusion: Surviving status is more important than in-and out-patient status in a patient's cluster membership based on blood test profile. This result suggests a plan to use both X-ray diagnosis and blood test results as a criterion to admit COVID-19 inpatients.
\end{abstract}

Keywords: COVID-19, outpatients, surviving, non-surviving, blood test results

\section{INTRODUCTION}

Since the end of 2019 and beginning of 2020, a new species of Coronavirus (called SARS-CoV-2), through personto-person respiratory transmission, caused a global pandemic (called COVID-19). The scope of reach is so widespread that COVID-19 is often compared to the 100year old $1918 \mathrm{H} 1 \mathrm{~N} 1$ virus pandemic $(1,2)$. COVID-19 also has a big impact on health system, as severe patients need oxygen, ventilation, and a bed in intensive care unit (ICU) for many days, if not weeks. On the other hand, many COVID-19 outpatients who have mild symptoms at the beginning may quickly lose body oxygen (hypoxia) and deteriorate towards potential death $(3,4)$.

Because the decision to put a COVID-19 patient in hospital (inpatient) is based on an apparent symptom, such as severity of pneumonia, we ask if blood test results may provide a further and better assessment on whether a patient should be admitted to the hospital or not. A Turkish cohort of more than 100 COVID-19 patients were collected from the Tokat State Hospital to address this question. Our cohort contains $56 \%$ outpatients and $44 \%$ inpatients-though not exactly half-half, provides a reasonable representation of both group of patients. Previously, there are other publications to characterize the demographic and symptomatic differences between the inpatients and outpatients (5). Although we also have demographic information (e.g. age, gender), the focus of this paper is on blood test measurement based characterization of COVID-19 patients (6). 
There have been other Turkish COVID-19 datasets being analyzed, such as (7-13). However, none of these address the differences between the three groups, i.e., outpatients, surviving inpatients, and non-surviving inpatients. Our unique dataset can be used to simultaneously question the outpatient-inpatient distinction and surviving-deceased patient distinction.

\section{MATERIAL AND METHOD}

The COVID-19 patients were collected from the Tokat State Hospital. Ministry of Health permission and Tokat Gaziosmanpaşa University Ethics Committee permission were obtained (Date: 01.04.2021, Decision No: $83116987 / 377)$. All procedures were carried out in accordance with the ethical rules and the principles of the Declaration of Helsinki.

A patient was examined with X-ray tomography for pneumonia. Those with pneumonia are classified into three groups: light, moderate, and severe. Light pneumonia patients were sent home as outpatients. Moderate pneumonia patients were further examined with other diseases: those (1) with additional diseases and/or (2) with other risk factors were admitted to hospital as inpatients. Severe pneumonia patients were admitted to the hospital as inpatients.

Blood tests for outpatients were taken at the time when they came to the hospital. Blood test for inpatients were taken both at the time of arriving at hospital, or before receiving treatment in a following day. These nine blood test factors are included in this paper: C-reactive-protein (CRP), calcium, potassium, sodium, vitamin-D, white blood cell (WBC) count, lymphocyte cell count, hemoglobin (HGB) and neutrophil.

Dimension reduction: We use two methods for dimension reduction: (1) t-SNE (full name: t-distributed stochastic neighbor embedding) (14) and (2) UMAP (full name: uniform manifold approximation and projection) (15). One difference between these methods and the more traditional PCA (full name: principal component analysis) and MDS (full name: multi-dimensional scaling) is that different clusters are not linearly proportional to the actual dissimilarity between the clusters, but is reasonable spaced. When we only want to illustrate clusters, this feature becomes an advantage because we optimally use the graph space. Both t-SNE and UMAP are run by R (www.r-project.orh) packages: Rtsne and umap. In Rtsne, the default parameter values are used, including perplexity $=30$. It is the same for umap where we use the default setting, e.g., number of neighbor $=15$, and use of Euclidean distance. Both t-SNE and UMAP has a random component, so each run can be slightly different from another run. The scale and direction of the $x$ and $y$ axes of a t-SNE or UMAP plot do not have any meaning, and they are simply marked as (component) 1 and 2.

\section{Statistical Analyses}

All statistical analyses are carried out by $\mathrm{R}$ functions, include cor (correlation coefficient), cor.test (correlation test), anova (analysis of variance), t.test (t-test), wilcox.test (Wilcoxon test), $\operatorname{lm}$ (linear regression), $\operatorname{glm}(\cdots$, family="binomial") (logistic regression). The MAD (full name: median absolute deviate) is defined as $\mathrm{MAD}=$ median $(\operatorname{abs}(\mathrm{x}-$ mean $(\mathrm{x})))$, which can be considered as a non-parametric equivalence to standard deviation.

\section{RESULTS}

Basic statistics of the data (first order): All 76 outpatients survived, whereas 60 inpatients can be further split into two groups: $n=45$ who survived and $n=15$ who died. Table 1 shows the mean and standard deviation of age and nine blood test measures, as well as the gender distribution, in these three groups. Generally, the factor value of the surviving inpatient group is in-between the mean values of the other two groups. For example, mean value for the $\mathrm{C}$-reactive protein (CRP) of the surviving-inpatient group is 11.08 , whereas that for the outpatient group is 7.97 , and that for the deceased inpatient group is 61.7. We also see that this cohort of COVID-19 patients is mostly female.

Original data may not be completely characterized by the summary statistics. For example, one may think from the mean age that the patients are mostly middle-aged. In fact, there are three young patients of ages 1,5 (surviving inpatient), and 5 (outpatient). Also, if a factor's value does not follow a normal distribution, its mean value is not the middle-range value one would think. To illustrate this point, we calculate the median and median absolute deviation (MAD) of all factors in the three groups in Table 1. While for most factors, the median and mean are similar, for CRP in the first groups, median is much smaller than the mean.

Basic statistics of the data (second order): The secondorder statistic of the dataset is the correlation between two factors. Table 2 shows the Pearson correlation coefficient and the corresponding $\mathrm{p}$-value, and non-parametric Spearman correlation (and the corresponding $\mathrm{p}$-value). A $\mathrm{p}$-value smaller than 0.005 is considered to be significant (16-18) and is marked with boldface in Table 2.

At this 0.005 significance level, HBG is correlated with 7 other factors considering either one of the Pearson/ Spearman correlations; calcium is correlated with 6 other factors, age, WBC is correlated with 5 other factors each; etc. In Table 2, the vitamin-D factor is only correlated with gender (lower than female). However, it might be an artifact in data collection: inpatients were taking vitamin-D supplement when they were in hospital, which may increase their vitamin-D level. 


\begin{tabular}{|c|c|c|c|c|c|}
\hline \multicolumn{6}{|c|}{ Mean/standard deviation, median/MAD, of all factors in three different groups } \\
\hline \multirow[t]{2}{*}{ Factor } & Outpatient & Inpatient (survived) & Inpatient (deceased) & Inpatient & All \\
\hline & $\mathrm{n}=76$ & $\mathrm{n}=45$ & $\mathrm{n}=15$ & $\mathrm{n}=60$ & $\mathrm{n}=136$ \\
\hline Age (mean \pm sd) & $49.7 \pm 17.8$ & $53.98 \pm 23.3$ & $74.47 \pm 9.06$ & $59.1 \pm 22.46$ & $53.85 \pm 20.45$ \\
\hline$($ median \pm MAD) & $50.5 \pm 12.5$ & $58 \pm 18$ & $76 \pm 3$ & $69.5 \pm 11.5$ & $55.5 \pm 17.5$ \\
\hline female $\%$ & $68.4 \%$ & $75.6 \%$ & $73.3 \%$ & $75 \%$ & $72.4 \%$ \\
\hline \multirow{2}{*}{ C-reactive-protein (CRP) } & $7.97 \pm 20.19$ & $11.08 \pm 25.54$ & $61.7 \pm 70.81$ & $23.09 \pm 45.72$ & $14.58 \pm 34.49$ \\
\hline & $2.015 \pm 1.632$ & $3.27 \pm 2.27$ & $69.6 \pm 48.295$ & $3.87 \pm 3.218$ & $3.06 \pm 2.492$ \\
\hline \multirow{2}{*}{ Calcium (mg/dL) } & $9.34 \pm 0.68$ & $9.2 \pm 0.86$ & $7.44 \pm 0.62$ & $8.76 \pm 1.11$ & $9.08 \pm 0.94$ \\
\hline & $9.365 \pm 0.385$ & $9.21 \pm 0.36$ & $7.41 \pm 0.49$ & $8.99 \pm 0.725$ & $9.24 \pm 0.52$ \\
\hline \multirow{2}{*}{ Potassium } & $4.32 \pm 0.42$ & $4.35 \pm 0.51$ & $4.04 \pm 0.91$ & $4.27 \pm 0.64$ & $4.3 \pm 0.53$ \\
\hline & $4.225 \pm 0.25$ & $4.37 \pm 0.31$ & $3.71 \pm 0.36$ & $4.265 \pm 0.44$ & $4.23 \pm 0.315$ \\
\hline \multirow{2}{*}{ Sodium } & $139.58 \pm 4.42$ & $140.49 \pm 3.09$ & $147.38 \pm 8.15$ & $142.21 \pm 5.65$ & $140.74 \pm 5.15$ \\
\hline & $139.35 \pm 1.8$ & $140.7 \pm 2.3$ & $147.8 \pm 2.5$ & $141.35 \pm 3.35$ & $140.1 \pm 2.3$ \\
\hline \multirow{2}{*}{ Vitamin D } & $17.47 \pm 14.12$ & $15.35 \pm 10.56$ & $13.48 \pm 8.11$ & $14.88 \pm 9.97$ & $16.31 \pm 12.46$ \\
\hline & $14.135 \pm 6.425$ & $13.07 \pm 7.35$ & $12.4 \pm 5.42$ & $12.735 \pm 7.01$ & $13.985 \pm 6.73$ \\
\hline \multirow{2}{*}{$\begin{array}{l}\text { White blood cell (WBC) } \\
\left(10^{9} / \mathrm{L}\right)\end{array}$} & $6.63 \pm 2.24$ & $7.43 \pm 4.38$ & $13.55 \pm 5.13$ & $8.96 \pm 5.26$ & $7.66 \pm 4.03$ \\
\hline & $6.25 \pm 1.335$ & $6.37 \pm 1.81$ & $13.29 \pm 2.89$ & $7.115 \pm 2.42$ & $6.525 \pm 1.775$ \\
\hline \multirow{2}{*}{ Neutrophil } & $4.43 \pm 2.04$ & $5.14 \pm 4.25$ & $12.25 \pm 4.83$ & $6.94 \pm 5.37$ & $5.53 \pm 4.04$ \\
\hline & $4.14 \pm 1.18$ & $3.85 \pm 1.615$ & $12.29 \pm 3.19$ & $5.43 \pm 2.71$ & $4.29 \pm 1.63$ \\
\hline \multirow{2}{*}{ Lymphocyte } & $1.57 \pm 0.7$ & $1.78 \pm 1.05$ & $0.71 \pm 0.37$ & $1.51 \pm 1.04$ & $1.54 \pm 0.86$ \\
\hline & $1.55 \pm 0.53$ & $1.69 \pm 0.345$ & $0.58 \pm 0.24$ & $1.4 \pm 0.49$ & $1.45 \pm 0.51$ \\
\hline \multirow{2}{*}{$\begin{array}{l}\text { Hemoglobin (HGB) } \\
(\mathrm{g} / \mathrm{dL})\end{array}$} & $12.88 \pm 1.71$ & $12.4 \pm 1.7$ & $9.55 \pm 1.54$ & $11.69 \pm 2.06$ & $12.35 \pm 1.96$ \\
\hline & $13.15 \pm 1.05$ & $12.4 \pm 0.8$ & $9.7 \pm 0.7$ & $11.85 \pm 1.45$ & $12.6 \pm 1.1$ \\
\hline
\end{tabular}

MAD: Median absolute deviate, (outpatients, surviving inpatients, and deceased/non-surviving inpatients). For each factor, there are two lines of summary statistics. The first line is mean \pm standard deviation; the second line is median \pm MAD. The last two columns are the summary statistics for inpatients (combining the two sub-inpatient groups), and for all (combining all three groups).

\section{Table 2. Correlation between any two factors. Two lines are used to show the second-order statistics}

\section{Correlation (Pearson/Spearman) and corresponding p-values of all pairs of factors}

\begin{tabular}{|c|c|c|c|c|c|c|c|c|c|c|}
\hline & Age & CRP & Cal & Pot & Sod & VitD & WBC & Neut & Lym & HGB \\
\hline \multirow[t]{2}{*}{ Sex } & $-.13(.12)$ & $-.16(.063)$ & $-.01(.94)$ & $0(.96)$ & $.09(.28)$ & $-.26(.0027)$ & $-.07(0.44)$ & $-.08(.37)$ & $.07(.4)$ & $-.35(3.2 \mathrm{e}-5)$ \\
\hline & $-.14(0.11)$ & $-.2(.022)$ & $.01(.89)$ & $-.01(.88)$ & $.18(.031)$ & $-.24(.0061)$ & $-.08(.35)$ & $-.1(.23)$ & $.03(.72)$ & $-.35(3.3 e-5)$ \\
\hline \multirow[t]{2}{*}{ Age } & - & $.27(.0018)$ & $-.49(1.7 e-9)$ & $.07(.44)$ & $.23(.0077)$ & $-.05(.55)$ & $.29(.00073)$ & $.35(3 e-5)$ & $-.35(3.8 e-5)$ & $-.17(.05)$ \\
\hline & - & $.22(.011)$ & $-.52(1.4 \mathrm{e}-10)$ & $.03(.75)$ & $.2(.019)$ & $-.04(.65)$ & $.24(.0056)$ & $.3(.00046)$ & $-.3(.00039)$ & $-.13(.14)$ \\
\hline \multirow[t]{2}{*}{ CRP } & & - & $-.18(.039)$ & $-.13(.14)$ & $.11(.19)$ & $.03(.76)$ & $.28(.0012)$ & $.3(.00046)$ & $-.12(.18)$ & $-.3(.00034)$ \\
\hline & & - & $-.09(.32)$ & $.01(.93)$ & $.14(.1)$ & $.11(.22)$ & $.15(.088)$ & $.12(.16)$ & $-.04(.64)$ & $-.12(.17)$ \\
\hline \multirow[t]{2}{*}{ Cal } & & & - & $.07(.44)$ & $-.3(.00041)$ & $.21(.017)$ & $-.45(2.8 \mathrm{e}-8)$ & $-.53(2.5 e-11)$ & $.38(4.7 e-6)$ & $.41(5.8 \mathrm{e}-7)$ \\
\hline & & & - & $.15(.09)$ & $-.17(.05)$ & $.17(.048)$ & $-.27(.0017)$ & $-.36(1.4 e-5)$ & $.39(2.9 e-6)$ & $.35(3.9 e-5)$ \\
\hline \multirow[t]{2}{*}{ Pot } & & & & - & $-.13(.12)$ & $.06(.52)$ & $0(.97)$ & $-.02(.83)$ & $.06(.51)$ & $.04(.63)$ \\
\hline & & & & - & $-.06(.52)$ & $.01(.91)$ & $.03(.69)$ & $.02(.86)$ & $.07(.4)$ & $.08(.36)$ \\
\hline \multirow[t]{2}{*}{ Sod } & & & & & - & $-.13(.15)$ & $.14(.1)$ & $.16(.056)$ & $-.13(.14)$ & $-.35(2.4 e-5)$ \\
\hline & & & & & - & $-.05(.57)$ & $.02(.81)$ & $0(.97)$ & $-.05(.58)$ & $-.31(.00023)$ \\
\hline \multirow[t]{2}{*}{ VitD } & & & & & & - & $-.01(.88)$ & $-.05(.57)$ & $.16(.062)$ & $.19(.025)$ \\
\hline & & & & & & - & $.06(.5)$ & $.05(.58)$ & $.12(.17)$ & $.16(.059)$ \\
\hline \multirow[t]{2}{*}{ WBC } & & & & & & & - & $.97(4.8 \mathrm{e}-84)$ & $.01(.87)$ & $-.32(.00017)$ \\
\hline & & & & & & & - & $.94(3.3 e-63)$ & $.05(.57)$ & $-.3(.00044)$ \\
\hline \multirow[t]{2}{*}{ Neut } & & & & & & & & - & $-.22(.0097)$ & $-.36(2.3 e-5)$ \\
\hline & & & & & & & & - & $-.23(.0071)$ & $-.3(.00044)$ \\
\hline \multirow[t]{2}{*}{ Lym } & & & & & & & & & - & $.19(.032)$ \\
\hline & & & & & & & & & - & $.26(.0023)$ \\
\hline
\end{tabular}


Clustering pattern for three groups by dimension reduction: Table 1 shows that the three groups: outpatients, surviving inpatients, and deceased inpatients potentially may have different blood test measures. To check this, we first consider each person as a point in the 9-dimensional space (for nine blood test variables, and note the age factor is not used), then project the points to low-dimensional space by a dimension-reduction technique. We use both t-SNE and UMAP, which are popular in single-cell expression data analysis as well as other fields $(14,15,19-23)$.

Before applying the dimension reduction techniques, there is another pre-processing option. We can either use the original dataset, or, forcing each factorsto contribute equally in the high-dimensional space by standardizing a factor (zero-mean and unit-variance). Actually, only the scaling part (forcing variance to be 1) is relevant, while shifting the mean to zero does not affect the dimension reduction result. Since CRP factor value does not follow a normal distribution, during pre-processing, CRP is log-transformed first before scaling it. Figure 1 shows four versions of the dimension reduction using t-SNE or UMAP, with or without standardization/scaling. Deceased samples are in red, surviving inpatients are in green, and outpatients are in blue. It can be seen that green and blue dots are not separated, whereas red dots are clustered, in particular for the standardized data.

Direct test of differences between three groups: To have a more direct proof on the differences between the three groups (outpatients, surviving inpatients, and deceased inpatients), three tests are carried out: (1) ANOVA which tests any difference between one or more groups from other groups; (2) t-test (or the non-parametric version: Wilcoxon test) between outpatients and surviving patients; (3) t-test (and Wilcoxon test) between the deceased inpatients and all surviving patients (both inpatients and outpatients). Gender is not a continuous value, but its proportion is not significant for any tests (p-values for (1) Fisher's test for 2-by-3; (2) Fisher's test between group-1 and 2; (3) Fisher's test between group-3 and combined groups 1 and 2 , are $0.73,0.53$, and 1 ).

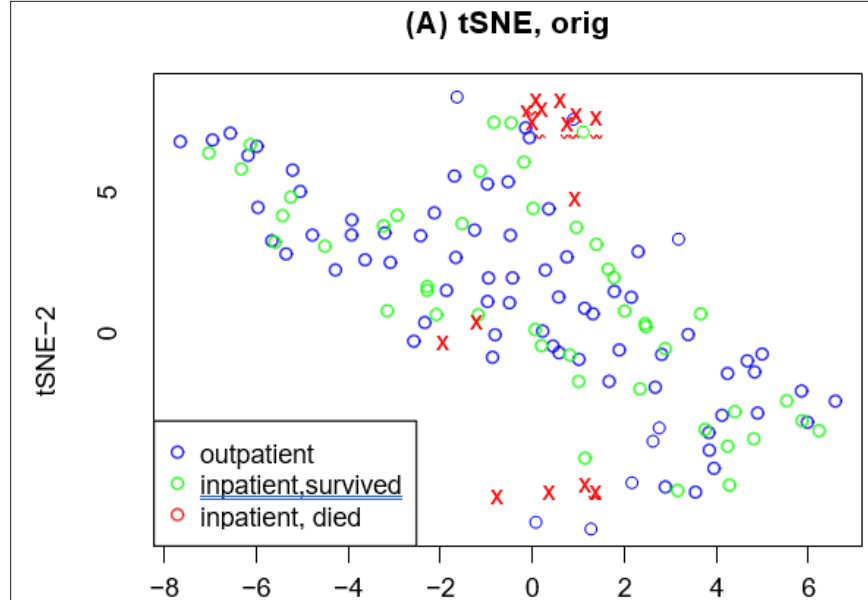

(C) UMAP, orig

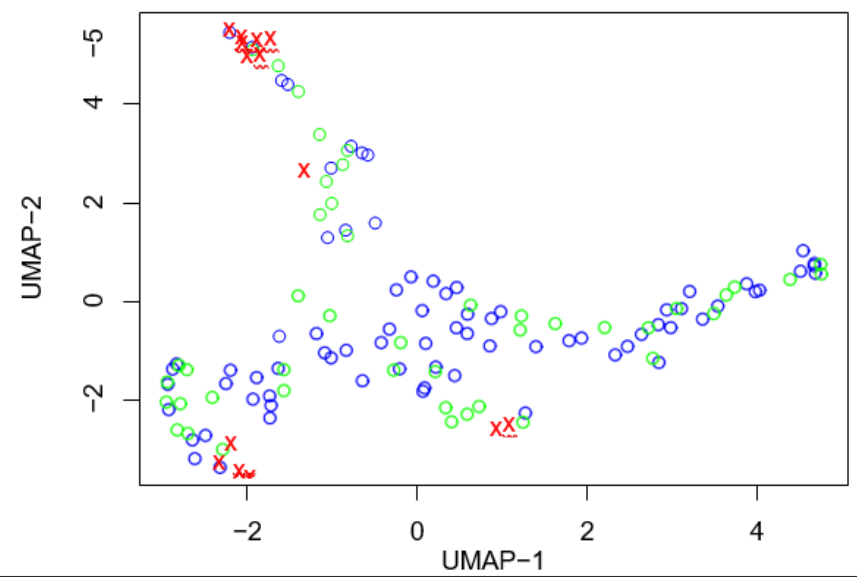

(B) tSNE, scaled

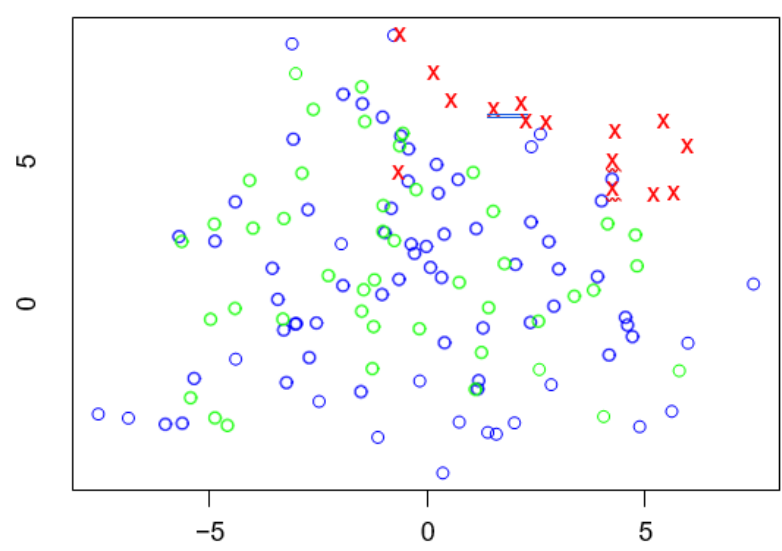

(D) UMAP, scaled

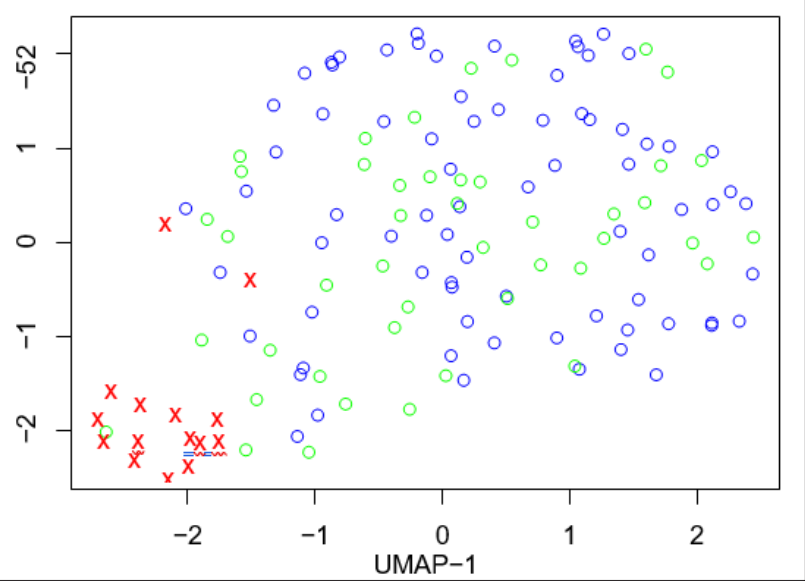

Figure 1. Projection of patient's blood test results from a 9-dimensional space to 2-dimension using two dimension-reduction techniques and/ or with variable scaling (also, CRP factor is log-transformed before scaling): (A) $t$-SNE using the original data; (B) $t$-SNE after each variable is standardized to zero-mean and unit-variance; (C) UMAP using the original data; (D) UMAP using the standardized data. Red dots represent deceased inpatients, green for surviving inpatients, and blue for outpatients (all survived).

tSNE: $t$-distributed stochastic neighbor embedding, UMAP: uniform manifold approximation and projection orig: original 
Table 3 shows the results of these three tests for nine blood test factors as well as age. For ANOVA test, all factors are significant at 0.005 level except potassium and vitamin-D. We have already mentioned that inpatients took vitamin-D supplement as soon as they were admitted to the hospital; this may provide a short-term boost to their vitamin-D level. Similarly, the potassium level can change quickly, within 5-6 hours. A more careful investigation would be to follow up the potassium level through time, and for this additional analysis with more data is needed.

\begin{tabular}{|lcccc|}
\hline \multicolumn{5}{|c|}{ Table 3. Various test results between groups for each factor } \\
t-test/Wilcoxin-test, ANOVA, conditional (on age) logistic \\
regression, p-values
\end{tabular}

Table 3 also shows that the significant ANOVA test results are all due to the difference in the deceased group, not between the inpatient (if they survive) and outpatient group. In other words, the separation between inpatients and outpatients is more artificial, whereas that between the surviving and non-surviving patients is more real. There are only two factors that show a potential trend (at the 0.1 level): sodium level is higher, and HGB is lower, for (surviving) inpatients than outpatients. Since these trends are not statistically significant at a level we feel confident, more data is needed to confirm or reject the observation.

To summarize our observations (Tables 1 and 3): when compared to the surviving patients whether they are inside or outside the hospital, deceased inpatients are older, higher CRP, white blood cell, neutrophil, and lower calcium, lymphocyte cell, hemoglobin. These differences collectively (excluding age) lead to a distinct group of non-surviving inpatients that are separated from surviving patients (both outpatients and inpatients), as shown in Figure 1.

Contribution of a factor to the surviving status conditional on age: Table 3 shows that almost all blood test factors are associated with the surviving status of a patient. However, the age is also associated with surviving status. Is a factor associated with surviving status because older patients tend to have different level of that factor? To address this question, we carry out a logistic regression: (death) (factor)+(age).
The last column in Table 3 shows the p-value for the conditional logistic regression where the effect of age is corrected. Although all p-values increase, the originally significant factors are still significant at 0.005 level. We conclude that these associated factors cannot be explained solely by the age differences in deceased and surviving patients.

Neutrophil-to-lymphocyte ratio (NLR) as a survival biomarker: Neutrophil-to-lymphocyte ratio (NLR) has been used as a biomarker for COVID-19 disease prognosis (24-27). Instead of using the NLR, we plot our data using only two factors: lymphocyte as $\mathrm{x}$-axis and neutrophil as $\mathrm{y}$-axis, in Figure 2A). It can be seen that similar to Figure 1B,D, Figure 2A also separates the deceased inpatients from both surviving inpatients and outpatients. A straight line going through the origin represents points with a constant NLR (the slope). We mark the NLR=10 line in Figure 2A, which reasonably separates red and green/ blue dots. With this line, three red dots are misclassified, or $3 / 15=0.2$ error rate; and 3-4 green/blue dots are misclassified, or $\sim 0.03$ error rate, including one surviving inpatient with a very large NLR value.

To add age information on topic of NLR, Figure 2B shows the scatter plot with age and NLR as two axes. Figure 2B essentially contains information from 3 variables, versus Figure $\mathbf{1}$ and Figure 2A which use information from 9 and 2 variables. Figure 2B clearly shows that NLR is high mostly within a limited age range (around age of 80 ). It also validates our previous result that older age itself is not $100 \%$ responsible for poor prognosis, but partially. Besides older age, other factors such as NLR also contribute greatly to the poor outcome of COVID-19.

Other biomarkers for prognosis: Table 2 shows that calcium level is correlated with both neutrophil and lymphocyte level. To show that calcium is also correlated with NLR, Figure $2 \mathrm{C}$ shows the scatter plot of the two, confirming that higher NLR is associated with low calcium level. Table 3 also shows that calcium is associated with age, which again can be shown directly, in Figure 2D. Similar observation was shown in the literature, e.g. (28).

Figure 3 shows the rest of factors that are significantly associated with the non-survival status according to Table 3: CRP, sodium, WBC, and HGB (neutrophil and lymphocyte are combined into a ratio in Figure $2 \mathrm{~B}$ and calcium in Figure 2D). All these plots show an interplay between these factors and age i.e., those non-surviving patients are predominately above certain old age; then, higher or lower level of these factors are, with various degrees, associated with the non-survival status (29). All these factors have been discussed in the COVID-19 literature: e.g., CRP, sodium and others, WBC, and HGB (30-33). 


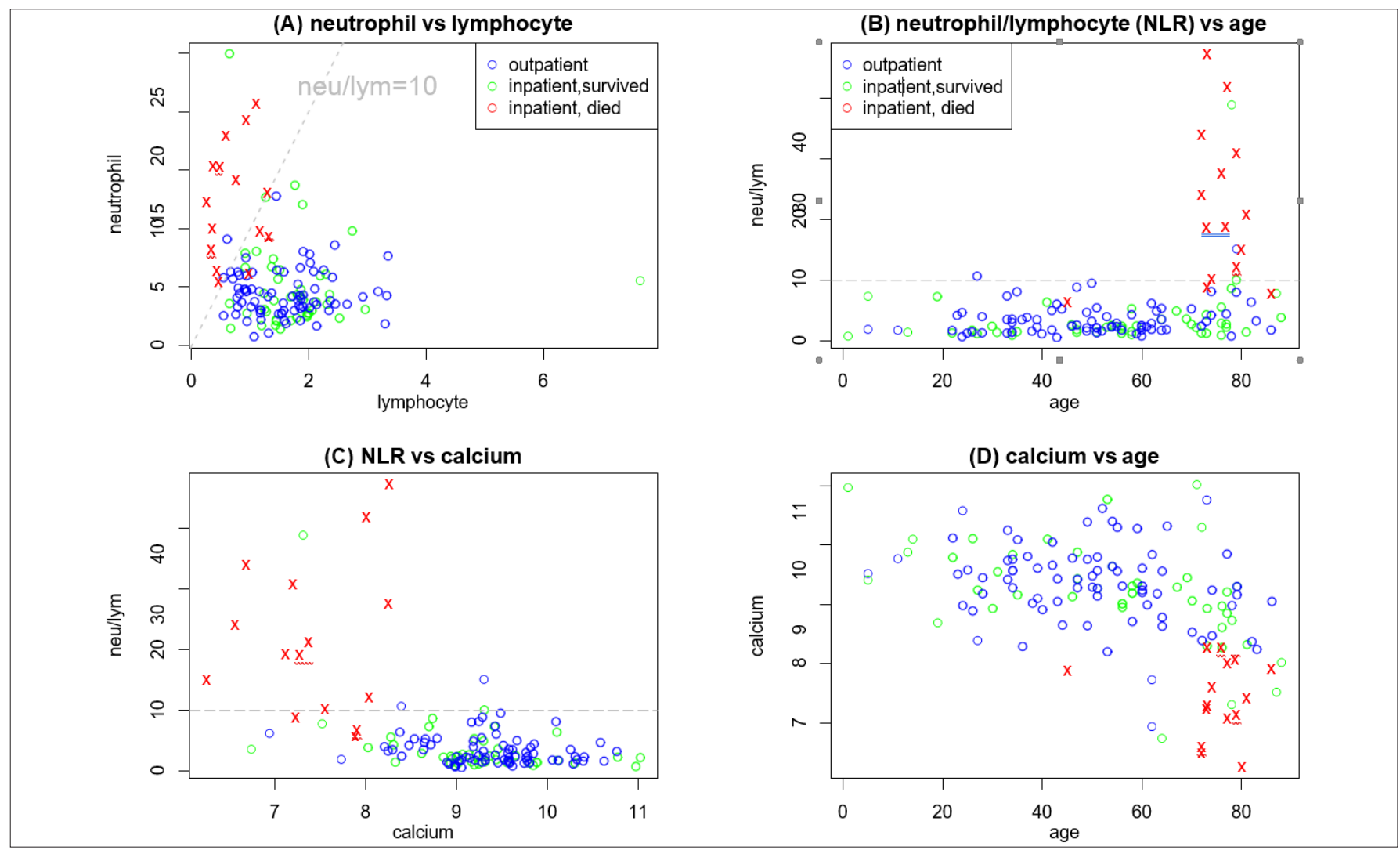

Figure 2. Scatter plots of (A) $x=$ lymphocyte, $y=$ neutrophil; (B) $x=$ age, $y=$ neutrophil/lymphocyte ratio (NLR); (C) $x=$ calcium, $y=N L R ;(D)$ $\mathrm{x}=$ age, $\mathrm{y}=$ calcium. Samples in three different groups are marked with different colors: red for non-surviving inpatients, green for surviving inpatients, and blue for outpatients. NLR: neutrophil-to-lymphocyte ratio

(A) CRP vs age

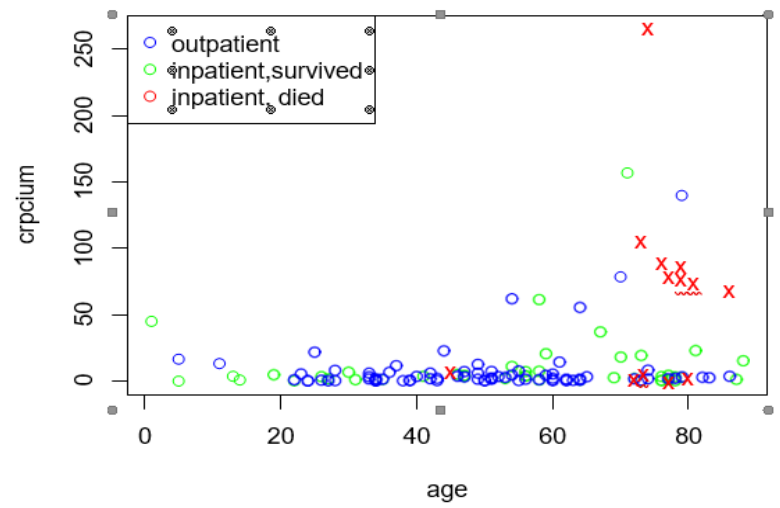

(C) WBC vs age

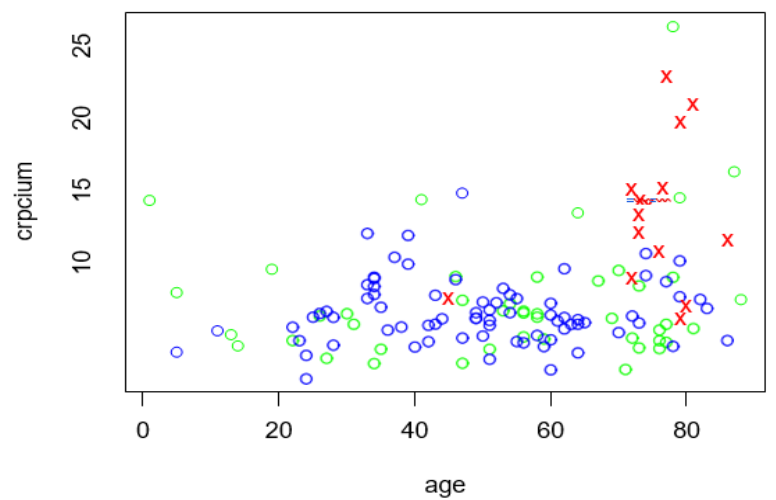

(B) sodium vs age

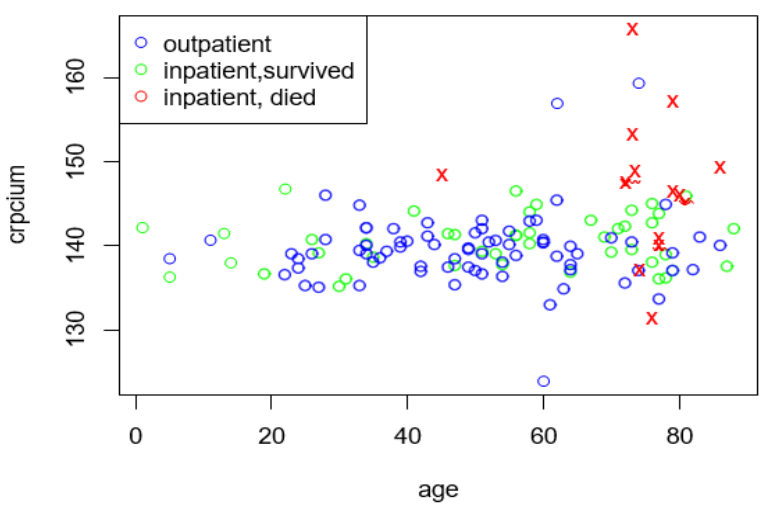

(D) HGB vs age

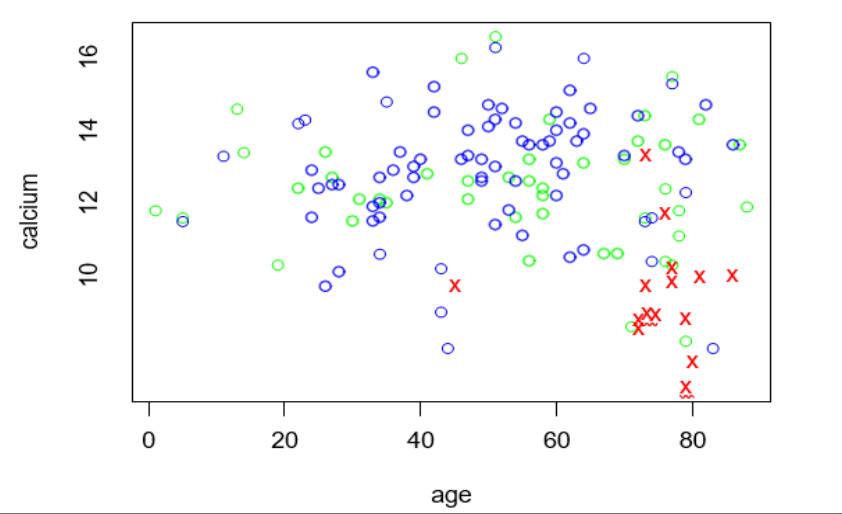

Figure 3. Scatter plots of factors over age: (A) CRP; (B) sodium; (C) white blood cell (WBC); (D) hemoglobin (HGB). Similar to Figure 2, red, green, blue represents deceased inpatients, survived inpatients, and outpatients. CRP: C-reactive-protein WBC: White blood cell, HGB: Hemoglobin 


\section{DISCUSSION}

It is common to project a multi-factor (high-dimensional) dataset to a two-dimensional plane to examine if there are clusters and sub-clusters. However, it is less discussed in the literature whether the data needs a preprocessing. In particular, whether each factor/variable should contribute equally to the high-dimensional distance between samples is a crucial consideration in preprocessing. To standardize factors, i.e., to make each factor to have zeromean and unit-variance, would treat all factors equally. Closely related to issue of standardization, for nonnormal distributions such as exponential or one-sided decaying distributions, the two parameters of mean and variance do not really characterize the distribution well, and it is desirable to transform the variable (e.g. logtransformation) to make the distribution normal-like.

Figure 1 shows that standardization (not the zero-mean part, but the unit-variance and some log-transformation the nine blood test measurement factors part) leads to a much better separation of the deceased samples (Figure 1B,D) compared to those without the standardization (Figure 1A,C). From our example, we recommend the standardization preprocessing step when multiple factors are considered jointly.

Our results show that among patients older than certain age, non-surviving inpatients have higher neutrophil level, lower lymphocyte level (higher NLR), lower calcium level, higher CRP, sodium, WBC, and lower HGB level, than the surviving patients; whether these are inpatients or outpatients. Comparing the two associated factors, CRP and HGB, there had been reports linking CRP level to severity of COVID-19 disease, and discussion on how anemia affects the quality of life in elder COVID-19 patients (34-36). Interestingly, Lippi et al pointed out that hemoglobin level may decrease in severe COVID-19 patients but the cause-effect direction is unclear (37).

In addition to CRP and HGB, calcium, potassium, sodium, WBC, neutrophil, lymphocyte cell count and Vitamin-D are extensively discussed in the literature (3850). All of these factors except Vitamin D and potassium show significant differences between non-surviving and surviving patients in Table 3 and are discussed in the literature, which confirm our findings and indicate dysregulation in COVID-19 patients.

The fact that surviving inpatients share more blood test resultsin common with outpatientsthan thenon-surviving inpatients has direct implication to patient management. Outpatients should carry out self-risk assessment (www. uptodate.com/contents/covid-19-outpatient-evaluationand-management-in-adults), and use cheap repurposing drugs. The treatment of outpatients has been evolving constantly. Patients visiting a hospital will go through medical examination to determine if they are in enough danger to stay in hospital. The main conclusion and lesson from this paper is that it is beneficial to not just use chest X-ray diagnosis alone, but also use blood test results, in screening COVID-19 patients for risk.

\section{ETHICAL DECLARATIONS}

Ethics Committee Approval: Ministry of Health permission and ethics committee permission at the Tokat Gaziosmanpasa University were obtained (Date: 01.04.2021, Decision No: 83116987/377).

Informed Consent: Written informed consent was obtained from all participants who participated in this study.

Referee Evaluation Process: Externally peer-reviewed.

Conflict of Interest Statement: The authors have no conflicts of interest to declare.

Financial Disclosure: The authors declared that this study has received no financial support.

Author Contributions: All of the authors declare that they have all participated in the design, execution, and analysis of the paper and that they have approved the final version.

\section{REFERENCES}

1. Taubenberger JK and Morens DM. The 1918 influenza pandemic and its legacy. Cold Spring Harb Persp Med 2020; 10: a038695.

2. Van Doren TP. The 1918 influenza pandemic has lessons for COVID-19: an anthropology student perspective. Am J Pub Health 2021; 111:79-80.

3. Chandra A, Chakraborty U, Pal J, Karmakar P. Silent hypoxia: a frequently overlooked clinical entity in patients with COVID-19. BMJ Case Rep 2020; 13: e237207.

4. Wilkerson RG, Adler JD, Shah NG, Brown R. Silent hypoxia: A harbinger of clinical deterioration in patients with COVID-19. Am J Emerg Med 2020; 38: 2243.e5-2234.e6.

5. Tenforde MW, Rose EB, Lindsell CJ, et al. Characteristics of adult outpatients and inpatients with COVID-19-11 Academic Medical Centers, United States, March -May 2020, Morb. Mortal Wkly Rep 2020; 69: 841-6.

6. Keshavarz M, Tavakoli A, Zanganeh S, et al. Clinical characteristics of outpatients and inpatients with COVID-19 in Bushehr: a report from the south of Iran. Future Med 2021; 16: 99-106.

7. Korkmaz MF, Türe E, Dorum BA, Kiliç ZB. The epidemiological and clinical characteristic of 81children with COVID-19 in a pandemic hospital in Turkey: an observational cohort study. J Korean Med Sci 2020; 35: e236.

8. Medetalibeyoglu A, Senkal N, Kose M, et al. Older adults hospitalized with COVID-19: clinical characteristics and early outcomes from a single center in Istanbul, Turkey. J Nutri Health Aging 2020; 24: 928-37.

9. Oncel MY, Akın IM, Kanburoglu MK, et al. A multicenter study on epidemiological and clinical characteristics of 125 newborns born to women infected with COVID-19 by Turkish Neonatal Society. Euro J Pediat 2020; 180: 733-42.

10. Kocayiğit H, Süner KÖ, Tomak Y, et al. Characteristics and outcomes of critically ill patients with COVID-19 in Sakarya, Turkey: a single center cohort study. Turk J Med Sci 2021; 51: 4407. 
11. Altunok ES, Alkan M, Kamat S, et al. Clinical characteristics of adult patients hospitalized with laboratory-confirmed COVID-19 pneumonia. J Infect Chemo 2021; 27: 306-11.

12. Satış H, Özger HS, Yildiz PA, et al. Prognostic value of interleukin-18 and its association with other inflammatory markers and disease severity in COVID-19. Cytokine 2021; 137: 155-302.

13. Harbalio `glu H, GençÖ, Yildirim A. 3 predictors of hospitalization patients with coronavirus (COVID-19): old age, lactate dehydrogenase and neutrophil/lymphocyte ratio. Pamukkale Tip Derg 2021; 14: 57-62.

14. Van der Maaten LJ and Hinton GE. Visualizing data using t-SNE. J. Machine Learning Res 2008; 9: 2575-605.

15.McInnes L, Healy J, Saul N, Grossberger L. UMAP: uniform manifold approximation and projection. J Open Source Software 2018; 3: 861.

16. Colquhoun D. The reproducibility of research and the misinterpretation of p-values. Royal Soc Open Sci 2017; 4: 171085.

17. Ioannidis JPA. The proposal to lower P value thresholds to. 005. JAMA 2018; 319: 1429-30.

18.Li W, Shih A, Freudenberg-Hua Y, Fury W, Yang Y. Beyond standard pipeline and $\mathrm{p}<0.05$ in pathway enrichment analyses. Comp Biol Chem 2021; 92: 107455.

19. Amir ED, Davis KL, Tadmor MD, et al. viSNE enables visualization of high dimensional single-cell data and reveals phenotypic heterogeneity of leukemia. Nature Biotech 2013; 31: 545-52.

20.Becht E, McInnes L, Healy J, et al. Dimensionality reduction for visualizing single-cell data using UMAP. Nature Biotech 2019; 37 38-44.

21.Li W, Cerise JE, Yang Y, Han H. Application of t-SNE to human genetic data. J Bioinfo Comp Biol 2017; 15: 1750017.

22.Li W, Freudenberg J, Freudenberg J. Alignment-free approaches for predicting novel Nuclear Mitochon-drial Segments (NUMTs) in the human genome. Gene 2019; 691: 141-52.

23. Ulgen A, Gürkut Ö, Li W. Potential predictive factors for breast cancer subtypes from a North Cyprus cohort analysis. Cyprus J Med Sci 2020; 5: 339-49.

24.Liu J, Liu Y, Xiang P, et al. Neutrophil to lymphocyte ratio predicts critical illness patients with 2019 coronavirus disease in the early stage. J Transl Med 2020; 18: 206.

25. Ponti G, Maccaferri M, Ruini C, Tomasi A, Ozben T. Biomarkers associated with COVID-19 disease progression. Crit Rev Clin Lab Sci 2020; 57: 389-99.

26. Ye W, Chen G, Li X, et al. Dynamic changes of D-dimer and neutrophil-lymphocyte count ratio as prognostic biomarkers in COVID-19. Resp Res 2020; 21: 169.

27. Moradi EV, Teimouri A, Rezaee R, et al. Increased age, neutrophilto-lymphocyte ratio (NLR) and white blood cells count are associated with higher COVID-19 mortality. Am J Emerg Med 2021; 40: 11-4.

28. Sun JK, Zhang WH, Zou L, et al. Serum calcium as a biomarker of clinical severity and prognosis in patients with coronavirus disease 2019. Aging 2020; 12: 11287-95.

29. Lim JP, Low KYH, Lin NJJ, et al. Predictors for development of critical illness amongst older adults with COVID-19: Beyond age to age-associated factors. Archives Gerontology and Geriatrics 2021; 94: 104331

30.Sahu BR, Kampa RK, Padhi A, Panda AK. C-reactive protein: a promising biomarker for poor prognosis in COVID-19 infection. Clinica Chimica Acta 2020; 509: 91-4.

31. Tezcan ME, Gokce GD, Sen N, Kaymak NZ, Ozer RS. Baseline electrolyte abnormalities would be related to poor prognosis in hospitalized coronavirus disease 2019 patients. New Microb New Infect 2020; 37: 100753.
32. Naoum FA, Zucareli Ruiz AL, de Oliveira Martin FH, Gomes Brito TH, Hassem V, de Lucca Oliveira MG. Diagnostic and prognostic utility of WBC counts and cell population data in patients with COVID19. Int J Lab Hematol 2021. Doi: 10.1111/ijlh.13395.

33. Algassim AA, Elghazaly AA, Alnahdi AS, et al. Prognostic significance of hemoglobin level and autoimmune hemolytic anemia in SARS-CoV-2 infection. Ann Hematol 2021; 100: 37-43.

34. Sharifpour M, Rangaraju S, Liu M, et al. C-Reactive protein as a prognostic indicator in hospitalized patients with COVID-19. PLoS One 2020; 15: e0242400.

35. Nemer DM, Wilner BR, Burkle A, et al. Clinical characteristics and outcomes of Non-ICU hospitalization for COVID-19 in a nonepicenter, centrally monitored healthcare system, J Hosp Med 2021; 16: 7-14.

36. Bergamaschi G, de Andreis FB, Aronico N, et al. Anemia in patients with COVID-19: pathogenesis and clinical significance, Clin Exp Med 2021. Doi: 10.1007/s10238-020-00679-4.

37.Lippi G and Mattiuzzi C. Hemoglobin value may be decreased in patients with severe coronavirus disease 2019. Hematol Transfus Cell Ther 2020; 42: 116-7.

38. Zhang L, Yan X, Fan Q, et al. D-dimer levels on admission to predict in-hospital mortality in patients with COVID-19. J Thromb Haemost 2020; 18: 1324-9.

39. Hachim MY, Hachim IY, Naeem KB, Hannawi H, Al Salmi I, Hannawi S. D-dimer, troponin, and urea level at presentation with COVID-19 can predict ICU admission: a single centered study. Front Med 2020; 7: 949.

40. Ceriello A. Hyperglycemia and COVID-19: What was known and what is really new? Diabetes Res Clin Pract 2020; 167: 108383.

41. Di Filippo L, Formenti AM, Rovere-Querini P, et al. Hypocalcemia is highly prevalent and predicts hospitalization in patients with COVID-19. Endocrine 2020; 68: 475-8.

42. Benedetti C, Waldman M, Zaza G, Riella LV, Cravedi P. COVID-19 and the kidneys: an update. Front Med 2020; 7: 423.

43. Chen D, Li X, Song Q, et al. Assessment of hypokalemia and clinical characteristics in patients with coronavirus disease 2019 in Wenzhou, China, JAMA Netw Open 2020; 3: e2011122.

44. Tzoulis P, Waung JA, Bagkeris E, et al. Dysnatremia is a predictor for morbidity and mortality in hospitalized patients with COVID-19. J Clin Endo Metab 2021. Doi: 10.1210/clinem/dgab107

45. Anurag A, Jha PK, Kumar A. Differential white blood cell count in the COVID-19: A cross-sectional study of 148 patients. Diabetes Metab Syndr 2020; 14: 2099-102.

46. Aschenbrenner AC, Mouktaroudi M, Krmer B, et al. German COVID-19 Omics Initiative (DeCOI). Disease severity-specific neutrophil signatures in blood transcriptomes stratify COVID-19 patients. Genome Biol 2020; 13: 1-25.

47. Huang I and Pranata R. Lymphopenia in severe coronavirus disease-2019 (COVID-19): systematic review and meta-analysis. J Intensive Care 2020; 8: 1-10.

48. Liu J, Li H, Luo M, et al. Lymphopenia predicted illness severity and recovery in patients with COVID-19: a single-center, retrospective study, PLoS ONE 2020; 15: e0241659.

49. Li X, Liu C, Mao Z, Xiao M, Wang L, Qi S. Predictive values of neutrophil-to-lymphocyte ratio on disease severity and mortality in COVID-19 patients: a systematic review and meta-analysis. Crit Care 2020; 24: 1-10

50. Simadibrata DM, Pandhita BAW, Ananta ME, Tango T. Plateletto-lymphocyte ratio, a novel biomarker to predict the severity of COVID-19 patients: A systematic review and meta-analysis. J Intensive Care Soc 2020; 1751143720969587. 